\title{
UN TEMA DE GAOS: ABSTRACCIÓN $Y$ VERDAD
}

Me propongo exponer aquí en líneas generales ciertos planteamientos teóricos de Gaos que permiten ver bien la articulación interna de su pensamiento, pues reiteran, desde ángulos diversos, convicciones básicas suyas y muy representativas de su modo de filosofar. Tuve oportunidad de discutir largamente con él estos planteamientos cuando seguí su seminario de El Colegio de México, en especial con ocasión de un muy incipiente trabajo crítico mío que él, a fuer de maestro concienzudo y devoto, analizó minuciosamente y luego comentó en notas escritas que conservo y que reproduciré más adelante.

El tema del debate era el origen de la validez a priori de ciertos enunciados cognoscitivos y, correlativamente, el de los grados de verdad y certeza que corresponden a las diversas ciencias y formas del saber. Unos pocos hechos, por todos reconocidos, daban la base de la argumentación y también eran fuente del problema que habia que resolver. Helos aquí: a) la validez universal y necesaria de los conocimientos matemáticos, cuyos enunciados pueden ser considerados apriorísticos por antonomasia; b) la endeblez y la inestabilidad epistemológicas de los enunciados filosóficos, reflejadas sobre todo en las rectificaciones y cancelaciones mutuas y constantes de los sistemas (decisivas en el caso de la metafísica, pero innegables también en otras disciplinas filosóficas, salvo la lógica); y c) la diferencia de grado en la validez de los conocimientos de la física y las demás ciencias naturales, de un lado, y de las ciencias sociales o espirituales, de otro, diferencia que obra en favor de la cientificidad de las primeras.

Situándose en el horizonte de la fenomenología pero aceptando las principales tesis sobre la lógica y el conocimiento científico que procedían del primer Wittgenstein y de los filósofos del Círculo de Viena, mi trabajo atendia al papel que desempeñan varios factores en la diferenciación del valor epistemológico de las ciencias y otras formas del saber y, en general, de cualquier sistema de enunciados con pretensión cognoscitiva. Conviene recordar los puntos que resaltaba, aunque sean banales, a fin de tener claro el término polémico de Gaos y lo que él no aceptaba o no consideraba importante en el tratamiento de la cuestión debatida.

En primer lugar llamaba yo la atención sobre la manera en que se constituyen los objetos intencionales de las aserciones científicas y filosóficas, haciendo resaltar el hecho de que en un caso el papel principal es desempeñado por la postulación, con la que se articula la deducción, mientras que en otros 
casos lo importante es la observación y la comprobación con referencia existencial. Se delineaba así la oposición entre las ciencias matemáticas y las ciencias positivas como dos grupos fundamentales de conocimientos.

Esta oposición se conectaba con otra muy familiar a Gaos por su temprana vinculación con el pensamiento de Husserl, la oposición representada por la pareja inmanencia-trascendencia. Las ciencias matemáticas o matematizables trabajan con lo que podemos llamar, husserlianamente, puros nóemas, mientras que las positivas empíricas remiten a trascendencias existenciales, de tal manera que para la validación de los enunciados de los primeros basta una inspección de la conformación de sus términos intencionales como tales, mientras que en el caso de las segundas es preciso ir más allá de dichos términos.

Esto nos lleva a otro factor que era necesario subrayar y en torno al cual se centró el debate con Gaos. La diferencia entre el valor cognoscitivo de los sistemas matemáticos y el de los empíricos tiene que ver esencialmente con la analiticidad o el carácter sintético de los enunciados o cadenas proposicionales de las ciencias. En mi enfoque, la analiticidad era la clave de la aprioridad de las matemáticas y su ausencia mayor o menor la del carácter contingente o necesario, en sus grados respectivos, de las demás ciencias y formas dẹ saber. Las ciencias se podían ordenar, según esto, de acuerdo a este factor. Por lo que toca a las diferencias epistemológicas que era posible encontrar en el grupo de las disciplinas positivas, debían remitirse a diferencias en la pureza analítica de las formulaciones. Cuando este factor era constante, la explicación del contraste podía buscarse en las dificultades de acceso al objeto real, con los ingredientes de simplicidad o complejidad, fijeza o mutación, simbolización más o menos adecuada, mensurabilidad, etc., como causas concomitantes.

En resumen, mi trabajo sostenía que la validez a priori de los enunciados (matemáticos $u$ otros) se origina en el papel fundamental que desempeña la estructura analítica de los enunciados, operando sobre la base de la preeminencia de la postulación y la demostración y de la reducción al círculo de lo noemático. Si esos factores no operan, aunque el objeto se dé con plena claridad e inmediatez, aunque su consistencia tenga la transparencia de la abstracción cabal, no se alcanza la validez a priori.

Para Gaos en cambio, los factores constitutivos y de inmanencia no eran los decisivos, ni lo era la analiticidad. Lo decisivo para él era la abstracción. Validez e invalidez, subjetividad y objetividad del conocimiento dependian, a juicio suyo, del grado de abstracción con que las diferentes disciplinas toman sus objetos. Correspondientemente, la debilidad epistemológica y el ingrediente de subjetividad que muestran ciertos conocimientos es función de la mayor o menor materia que ofrecen sus objetos o que se ha tomado en consideración al estudiarlos. La intersubjetividad o su ausencia le vienen a los 
conocimientos de la presencia o ausencia de concreción en los objetos. $\mathrm{Y}$ esto es así porque las realidades abstractas, o el abstracto de las realidades, no apela a nada personal o individual; no compromete al sujeto concreto en su ser diferente; lo deja fuera de juego, indiferente y por tanto capaz de participar de lo que es común.

Con la intersubjetividad del conocimiento se funda la jerarquía de las ciencias. En la cima de la pirámide se encuentran las matemáticas porque sus objetos se dan como muy abstractos -más tarde Gaos escribirá en sus Confesiones profesionales: "Los sujetos que se abstraen de la totalidad de la realidad dada a cada uno de ellos menos de las formas geométricas que son parte de ella, para abstraerse precisamente en estas formas, son los geómetras"," lo cual vale para los matemáticos en general con sus objetos propios. Por esta razón, su validez es plenamente universal y necesaria. A continuación se sitúan las ciencias físicas y naturales. Están bastante cerca de las matemáticas por el rigor de sus formulaciones y explicaciones, pero el ingrediente material de sus objetos, o sea, la menor abstracción, impide a sus enunciados alcanzar el nivel epistemológico de las ciencias exactas. Finalmente - para no tocar el caso de la filosofía que debe ser tratado aparte-, las ciencias sociales y espirituales ven mermado el alcance y la necesidad de sus enunciados en la medida en que sus objetos propios son concretos y decididamente individuales. La intersubjetividad de las ciencias del hombre está, en consecuencia, muy lejos de la intersubjetividad matemática, no por la trascendencia existencial de sus contenidos cognoscitivos, ni por la ausencia de conexiones analíticas de sus enunciados, sino por la correspondiente abstracción mayor de sus objetos. La filosofía, en fin, que era tema muy caro a Gaos, se sitúa en las antípodas de las ciencias exactas pues su tema es inseparable de la realidad en su plena integración y de los hombres singulares que son el foco de la experiencia filosofante.

Este planteo, en sus diversos aspectos y consecuencias filosóficas, se encuentra muy precisamente formulado en las siguientes notas de Gaos:

1. La intersubjetividad de las ciencias no depende de la forma analítica que se ha dado o pueda darse a sus materias u objetos, sino de la abstracción de éstos.

2. Cuando dada una materia conformada analíticamente se apela a su intersubjetividad, ésta es de la forma no de la materia, o el objeto al que exclusivamente se apela es la forma. Ejemplo: 'El árbol de mi jardín es el árbol de mi jardín'.

3. La intersubjetividad de la ciencia cuyo objeto es la forma analítica misma depende de la abstracción, máxima, de su materia u objeto, que es la forma analitica misma de toda materia $u$ objeto (ente, ser) posible; esta forma, to-

I Confesiones profesionales, México, Tezontle, 1958, p. 11. 
mada como materia $u$ objeto puede conformarse, naturalmente, por medio de ella misma.

4. El objeto "objeto" en general es el más abstracto de todos. Si implica alguna referencia a algún sujeto, es exclusivamente a un sujeto no menos general o abstracto - de todo lo que pueda pluralizar los sujetos con sus perspectivas del mundo.

5. Si hay un mundo es porque la pluralidad de los sujetos individualmente irreductibles o absolutos tiene también una unidad parcialmente producto de la abstracción máxima: la unidad de todo es la unidad pura y simple. Cf. Hegel.

6. El dar la forma analítica a materias concretas no hace ciencias de estas materias. Los sectores de la cultura siguen siendo tan irreductibles, aunque se les dé la forma analítica. ${ }^{2}$

Tomando pie en estas observaciones críticas, veamos más de cerca algunos puntos fundamentales de la tesis del maestro español.

a) Los comentarios de Gaos ponen de manifiesto dos convicciones suyas muy arraigadas, a saber: que el objeto tiene una función decisiva en el carácter a priori de la validez de los enunciados, y que un tipo de objetividad determinado es el que da base a la aprioridad. Según esto, el fundamento de la validez del conocimiento no es inmanente a los enunciados sino que remite a los términos intencionales de éstos. Én todo caso, la estructura y caracter del objeto, reflejándose en el enunciado a través de la forma de los asertos, da al conocimiento su universalidad y su necesidad.

¿En qué consiste este tipo de objetividad, esta condición de los objetos gracias a la cual los enunciados son válidos a priori? Como sabemos, para Gaos la respuesta es simple y precisa: la abstracción. Aquí tocamos el núcleo de la epistemología y de la ontología de nuestro filósofo. La categoria de la abstracción es la clave del ser y del conocer y da cuenta tanto del valor de los conocimientos cuanto de la unidad o pluralidad de los existentes.

En este planteo influye decisivamente la formación fenomenológica y orteguiana de Gaos. Aunque no apela a la evidencia apodíctica o a la intuición eidética, en su tesis opera la teoría husserliana del cumplimiento de las ideas en la intuición y de la dación por escorzos de los objetos de nuestra experiencia, teoría que parafraseó Ortega en su perspectivismo, del que se nutrió también Gaos. La aprehensión de lo concreto es siempre parcial, deja residuos de intenciones no cumplidas. El objeto lleno se da necesaria e inevitablemente por escorzos, en perspectiva. Ahora bien, cuanto menos plena sea su entidad, cuanto más se acerque a una pura forma, mayor cabalidad de aprehensión hace posible, menos escorzos y visiones oblicuas se interponen entre el sujeto que conoce y la verdad. De alli la validez y la certeza máximas de los conocimientos que versan sobre objetos abstractos.

Se comprende entonces que la intersubjetividad que es propia de los enunciados válidos a priori no sea ajena, en el planteo de Gaos, a esta fun-

2 Noviembre de 1949 . 
ción de la abstracción, ya que, como vimos, cuanto más abstracto es un objeto menos referencias implica a un sujeto determinado. A lo más remite a un sujeto universal -cuasi-vacio- que se confunde con la intersubjetividad misma.

b) Aunque ha formado tradicionalmente parte de la filosofía, la lógica es una disciplina ejemplar por la necesidad y la universalidad de sus asertos y por la muy vasta intersubjetividad que, paralelamente, alcanzan sus conocimientos. Esto se explica en el planteo que aquí exponemos, porque la lógica tiene por tema la forma analítica de todos los conocimientos deductivos. Esta forma analítica como tal es una instancia que no tiene determinación alguna relativa a la materia de un ente o ser, tomado en particular. El principio de la abstracción sigue siendo pues la norma de- la aprioridad, inclusive en el caso de la propia analiticidad. Para Gaos, la lógica posee conocimientos apriorísticos no porque sus enunciados formulen conexiones analíticas o sean ellos mismos enunciados analíticos (cosa que se acepta de modo explícito al decir, como lo hace Gaos, que la forma analítica "tomada como materia $u$ objeto, puede.conformarse, naturalmente, por medio de ella misma"), sino porque la estructura analítica en cuanto tal es un objeto abstracto en grado máximo, es decir, despojado de todo elemento material o de concreción óntica. Con su carácter formal -entendido aquí simplemente como no-material, sin contenido determinado-, el objeto lógico alimenta la validez a priori del cuerpo sistemático de la disciplina, quedando un poco descartados como fuente de validez los elementos deductivos y analíticos del sistema.

La aprioridad fundada de este modo en la abstracción se extiende luego a todos los enunciados y conjuntos conceptuales que se conforman mediante la forma analítica, los cuales resultan también válidos a priori, pero ahora por analíticamente formulados. Sin embargo, Gaos era muy enfático en la afirmación de que en última instancia, esta estructura analítica se conecta con la aprioridad por la abstracción y no por su propia constitución, es decir, por la analiticidad.

c) La realidad es una solamente en cuanto se la toma sin atender a su materia concreta. Hay un mundo, o del mundo se puede predicar la unidad cabal, únicamente si se piensa en una instancia indiferenciada, ajena a las peculiaridades y diversidades de la existencia concreta.

Aquí también, para Gaos, el criterio decisivo es, como se observa, el de la abstracción. Quien piensa en plan abstracto, atendiendo a lo que es común o constante, a lo que se reitera en la existencia, y descarta todo lo demás, puede pensar un mundo y puede postular la unidad de la realidad. $Y$ en esta unidad indiferenciada de todo, que es una unidad total pero ideal - "la unidad de todo es la unidad pura y simple"-, puede lograr el acuerdo con todos los sujètos, la intersubjetividad, que es la perla fina del conoci- 
miento entendido como coincidencia de todos los hombres es la misma verdad. Quien abstrae, logra la unidad del mundo, pero se abstrae él mismo del mundo real, suyo, diverso.

En cambio, quien piensa en plan concreto, quien atiende a la materia objetiva, o a las materias innúmeras de las realidades, a la diversidad y la mutabilidad de las instancias existentes, ése no encuentra la unidad de la realidad, un mundo o el mundo como uno, pero piensa y vive lo real efectivo, no una instancia ideal. Porque no abstrae, no se abstrae o separa de nada y, sobre todo, de sí mismo. Encuentra por tanto la diversidad de la existencia fundada en la pluralidad de los sujetos, cada uno con su perspectiva, su mundo y su verdad, y él con la suya en primer y fundamental lugar. No podrá, pues, aspirar a la intersubjetividad, ni a la certeza apriorística, es decir, a ninguna forma de conocimiento entendido como coincidencia total de los hombres en la verdad una. Pero no por eso estará divorciado de la verdad y del conocimiento. Quien piensa en concreto no ha dejado de tener mundo y realidad, ni ha abandonado el terreno del cono cimiento, aunque se trate de otro mundo, de otra realidad, y de otro conocimiento.

d) Lo anterior hace ver claramente la situación de las ciencias histórica espirituales (sociales o culturales) en el enfoque de Gaos. Si son ciencias que atienden a lo concreto, es decir, si no se desentienden de la vida humana en lo que es propio y característico de ésta, entonces no pueden alcanzar la intersubjetividad de los enunciados válidos a priori. Sólo un saber de abstractos da pie a la coincidencia y a la prioridad, mientras que la realidad humana es plural y temporal. La existencia de las personas con nombre propio en que consiste la historia, o la realidad de las personas que viven instaladas en el fluir temporal y determinadas por su personalidad y sus vínculos mutuos, en suma, la historia, nunca es abstracta.

$Y$ no se puede remediar en nada esta situación que afecta a los sistemas de conocimientos histórico-culturales, de acuerdo a Gaos, porque el único recurso que parecerfa válido, la matematización por conformación analítica, no alcanzaría nunca a la materia del conocimiento, sino tan sólo a la forma, abstracta, del objeto. El contenido concreto de la vida, la existencia de los hombres en su singularidad y su sustancia diversificada y cambiante, quedarían irremediablemente afuera.

¿Significa esto negar a las ciencias históricas el título de formas del conocimiento? Gaos no lo cree. Para él se trata más bien de marcar una diferencia esencial entre dos formas de conocimiento o dos extremos polares de la serie de formas del conocimiento, a saber, la del conocimińto de lo plenamente abstracto y la del conocimiento de las singularidades concretas, oposición que coincide con la polaridad intersubjetividad-carácter personal del conocimiento. 
e) Pero el verdadero extremo de la serie por el lado del conocimiento concreto no son las ciencias (particulares) históricas. El cabo genuino es la filosofía. Como remate de estas reflexiones y de las implicaciones encerradas en las notas que comentamos, vuelve a aparecer una vieja idea de Gaos: la filosofía como confesión personal. Enlazando los dos extremos del argumento -y marcando de este modo la unidad interna de las convicciones de nuestro filósofo- podemos decir que, puesto que el saber a priori es saber de abstractos, la filosofía es y no puede menos de ser confesión personal, sin dejar de ser conocimiento. La filosofía está en el polo opuesto del conocimiento intersubjetivamente válido, de la verdad a priori en el modo de la matemática, porque pretende ser un saber de lo real en su más cabal y plena integridad, un saber totalitario no atenido a la pura forma sino a la entera y total materia. No puede por tanto ser indiferente a ninguna cosa, a ningún contenido entitativo, a ninguna variante existencial de las personas y los hechos y de sus articulaciones mutuas y concretas.

Puesto que el filósofo no abstrae nada, ni se abstrae en nada particular, sino en la totalidad (cf. Confesiones profesionales, p. 11), aquello que formula con la pretensión de verdad, los filosofemas, no pueden ser ajenos a su propia situación vital. Dicho de otro modo, lo que el filósofo afirma no tiene sentido sino en el contexto de su vida, porque incorpora su vida en el concreto de todo lo que existe o de todas las realidades, con su pluralidad irreductible, unificable de innúmeros modos. No será el suyo, por consiguiente, un saber del mundo uno, de la unidad (ideal) de la realidad, pero será, sin embargo, un saber, conocimiento de este mundo, de la multiplicidad de los modos de ser y devenir lo real y, por tanto, un saber que se expresa en términos teñidos de autobiografía, que revelan y acuñan lo que hay de propio e irrepetible en cada sujeto filosofante.

El objeto filosófico compromete así al sujeto, lo integra, lo presupone y reclama. Ratificando y radicalizando esta idea, en uno de sus últimos trabajos, dirá Gaos que tal objeto es "el más subjetivo de todos, el único absolutamente subjetivo, o en rigor, el único subjetivo, sin más -en una peculiar dialéctica de totalidad concreta y subjetividad, parcialidad abstracto-concreta e intersubjetividad, abstracción e intersubjetividad total..." в Y líneas adelante: "No sólo cada filósofo tiene su filosofía, sino que va haciéndola a lo largo de su vida, si no es que a lo largo de ésta tiene diversas filosofías, o cada filosofía es en conjunto, no sólo subjetiva sino momentánea, por intersubjetiva e intermomentánea que sea en partes de ella." 4

Afirmando el carácter irreductible del saber filosófico - $y$, en su nivel, del saber histórico-, Gaos quería dejar a salvo la verdad de la filosofía, la verdad del discurso filosófico, como histórica, personal, no gregaria, es decir,

3 De la filosofía, México, Fondo de Cultura Económica, Dianoia, 1962, p. 454 .

4 Id., pp. $454-455$. 
una verdad que, aunque no sea "universalmente válida, o verdad igualmente para todos",5 tiene genuina validez. En última instancia, en el reino del conocimiento, como en el del ser, para el maestro español quedaban frente a frente, como interlocutores mudos, el logos de la verdad abstracta, plenamente cierto por obra de su objeto indeterminado, y el logos de la verdad concreta, no menos cierto aunque por la razón exactamente contraria, por la singularidad cabal del hombre, de $m i$ persona en $m i$ mundo.

Augusto Salazar Bondy

UnIVERSidad de SAN MaRcos, Lima 